\title{
A photoconductive, miniature terahertz source
}

\author{
Richard K. Lai, Jiunn-Ren Hwang, Theodore B. Norris, and John F. Whitaker ${ }^{\text {a) }}$ \\ Center for Ultrafast Optical Science, University of Michigan, Ann Arbor, Michigan 48109-2099
}

(Received 14 January 1998; accepted for publication 13 April 1998)

\begin{abstract}
We discuss the performance of a micromachined, photoconductive terahertz emitter that is fabricated on low-temperature-grown GaAs. The device is mounted on a pair of single-mode optical fibers that allows the source to be freely positionable. A strong radiation burst is emitted due to the large magnetic moment created by the electrode. The emitter's small feature size of $300 \mu \mathrm{m}$ by 300 $\mu \mathrm{m}$ with a photoconductive switch area of $30 \mu \mathrm{m}$ by $30 \mu \mathrm{m}$ suggests its application for terahertz, time-domain, near-field spectroscopy and imaging. (C) 1998 American Institute of Physics.
\end{abstract}

[S0003-6951(98)00724-4]

Sources for freely propagating terahertz $(\mathrm{THz})$ radiation are important for spectroscopic studies of phenomena such as carrier and exciton dynamics in semiconductor structures and for $\mathrm{THz}$ emission from phonons and electron cyclotron oscillations. ${ }^{1-4}$ Prospects for useful imaging applications using $\mathrm{THz}$ beams have also recently become evident. ${ }^{5}$ One of the more common $\mathrm{THz}$ generation and detection systems consists of a pair of photoconductive switches defined by coplanar transmission lines on low-temperature-grown GaAs (LT-GaAs) or silicon-on-sapphire (SOS) substrates. One device then acts as an emitter and the other as a detector. ${ }^{6}$ The picosecond electric dipoles necessary for $\mathrm{THz}$ generation are created by electrically shorting the coplanar lines with an ultrashort laser pulse. As a result, the accelerating electrons between the lines emanate a pulse of broadband radiation into the substrate, with spectral content extending into the $\mathrm{THz}$ regime. The $\mathrm{THz}$ pulse duration is determined by the electron transport and screening dynamics, which is typically on the order of a picosecond. High-resistivity-silicon or sapphire lenses and parabolic mirrors are used for collimating and focusing the THz radiation. The output radiation is focused onto the detector, where another ultrashort-laser pulse train, time delayed with respect to the laser emitter pulse, is employed for photoconductive gating. Focusing of the radiation onto a sample of interest results in a spatial resolution of several hundred microns limited by diffraction. Also, the focused beam diameter can be frequency dependent over a large $\mathrm{THz}$ bandwidth. On the other hand, a flexible, freely positionable point source in contact with a sample would have a resolution of tens of microns, determined by the size of the photoconductive switch. In this letter, we describe the fabrication and mechanism of the $\mathrm{THz}$ generation for such an emitter.

Recently, an epitaxial-layer, fiber-mounted photoconductive (PC) probe coupled to a JFET source-follower amplifier was developed to measure guided picosecond electrical signals with high sensitivity. ${ }^{7}$ We now describe a micromachined $\mathrm{THz}$ emitter that is produced using similar fabrication techniques as those employed for the PC probe. The device, mounted on a pair of single-mode optical fibers,

a)Electronic mail: whitaker@engin.umich.edu has been used to launch free-space $\mathrm{THz}$ signals from both electric and magnetic dipoles.

The emitter was fabricated on a GaAs layer grown by molecular beam epitaxy at $240{ }^{\circ} \mathrm{C}$ and annealed in situ at $600{ }^{\circ} \mathrm{C}$ for $10 \mathrm{~min}$. The carrier relaxation of the LT-GaAs was measured by pump-probe transient absorption to be about 2 ps. In order to isolate the $1-\mu \mathrm{m}$-thick LT-GaAs layer and produce the free-standing PC device, mesa etching, titanium/gold metallization patterning, and backside etching have been used. ${ }^{7,8}$ The resulting emitter consisted of a 500/ $3000 \AA$, Ti/Au, metal-semiconductor-metal (MSM) interdigitated structure with $2 \mu \mathrm{m}$ finger size and spacing resulting in a switch area of $30 \mu \mathrm{m}$ by $30 \mu \mathrm{m}$. This MSM structure acted as the PC switch (Fig. 1). Two gold electrodes provided the bias and ground connections across the PC switch.

A pair of single-mode optical fibers were glued onto the emitter using ultraviolet-curing cement. One of the fibers was polished with a $45^{\circ}$ bevel to allow the guided laser pulses to internally reflect off the core surface to the backside of the emitter and into the MSM photoconductive switch. The other fiber provided only mechanical stability. Silver epoxy was placed along each of the fibers and onto the elec-

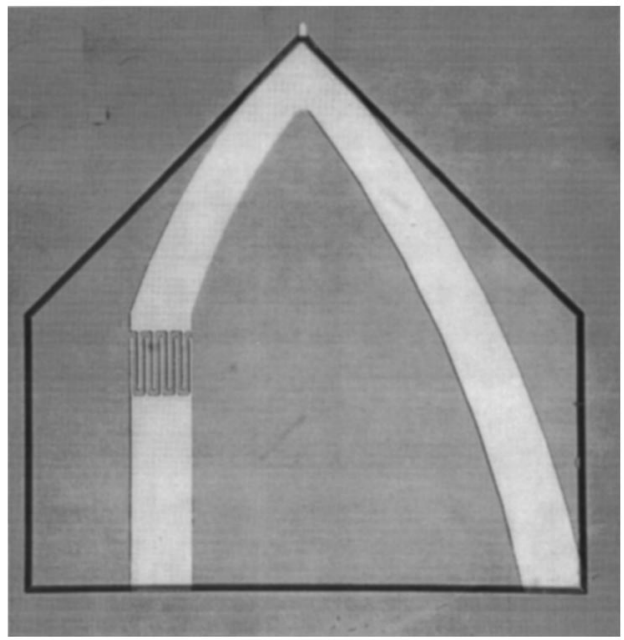

FIG. 1. Photomicrograph of the THz emitter before liftoff from the GaAs substrate. The emitter is $300 \mu \mathrm{m}$ by $300 \mu \mathrm{m}$ by $1 \mu \mathrm{m}$ and consists of a Ti/Au interdigital MSM switch with finger size and spacing of $2 \mu \mathrm{m}$. The switch area is $30 \mu \mathrm{m}$ by $30 \mu \mathrm{m}$. The left electrode was connected to a dc voltage source while the right electrode was connected to ground. 


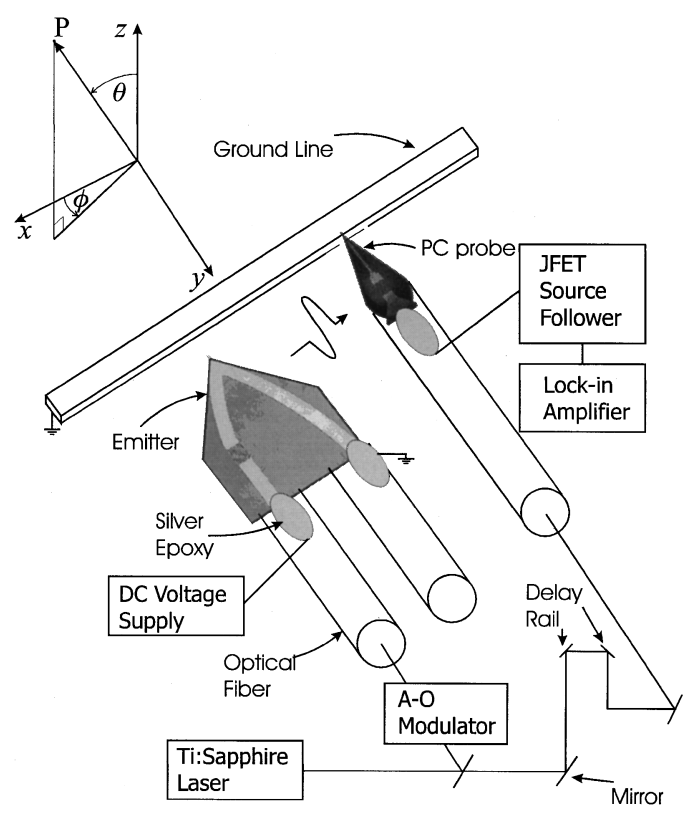

FIG. 2. Experimental setup with the fiber mounted emitter and PC probe acting as a terahertz receiver. The PC switch of the emitter was chosen as the origin of the coordinate axis (top of figure).

trodes as a means for electrical connections. The fibers were mounted onto a mechanical stage for positioning.

We measured the $\mathrm{THz}$ radiation from the emitter using a PC probe as the receiver (Fig. 2). A mode-locked Ti:sapphire laser operating at $810 \mathrm{~nm}$ wavelength with a repetition rate of $82 \mathrm{MHz}$ and a $100 \mathrm{fs}$ pulse duration was used to provide the optical excitation pulses in this setup. The beam was split into two, with one pulse train (variably delayed with respect to the other) modulated by an acousto-optic Bragg cell at a frequency of $10 \mathrm{kHz}$ and coupled into the emitter's singlemode fiber. The power of this beam was estimated to be about $5 \mathrm{~mW}$. The second beam was used to trigger the PC probe sampling head, which was positioned with its tip in contact with a long, narrow grounded line. The output of the PC probe was connected through a source follower to a lock-in amplifier. The tip of the probe was brought into contact with a long, narrow, ground line. The emitter electrode leading to the PC switch was biased with a $5 \mathrm{~V}$ dc supply, while the other electrode on the emitter was grounded. The relative electric field of the radiated signal was measured by reading the lock-in amplifier at different delay times between the two laser pulse trains.

The measured signals contain contributions from both electric and magnetic dipoles. In certain directions (i.e., in the plane) the magnetic component dominates. For instance, Fig. 3(a) shows a temporally resolved waveform generated by the emitter when it was located $500 \mu \mathrm{m}$ away from the PC probe in the $x$ direction (Fig. 2). The strong lateral radiation of the new emitter is unusual for $\mathrm{THz}$ signal sources, which typically radiate a signal from a transient electric dipole created between the two electrodes of a PC switch. In Fig. 3(b) dipoles are formed between the tips of the interdigitated fingers and the bases of the other electrode. However, most of the radiation detected in Fig. 3(a) is produced by a transient magnetic moment because the semilooping struc-

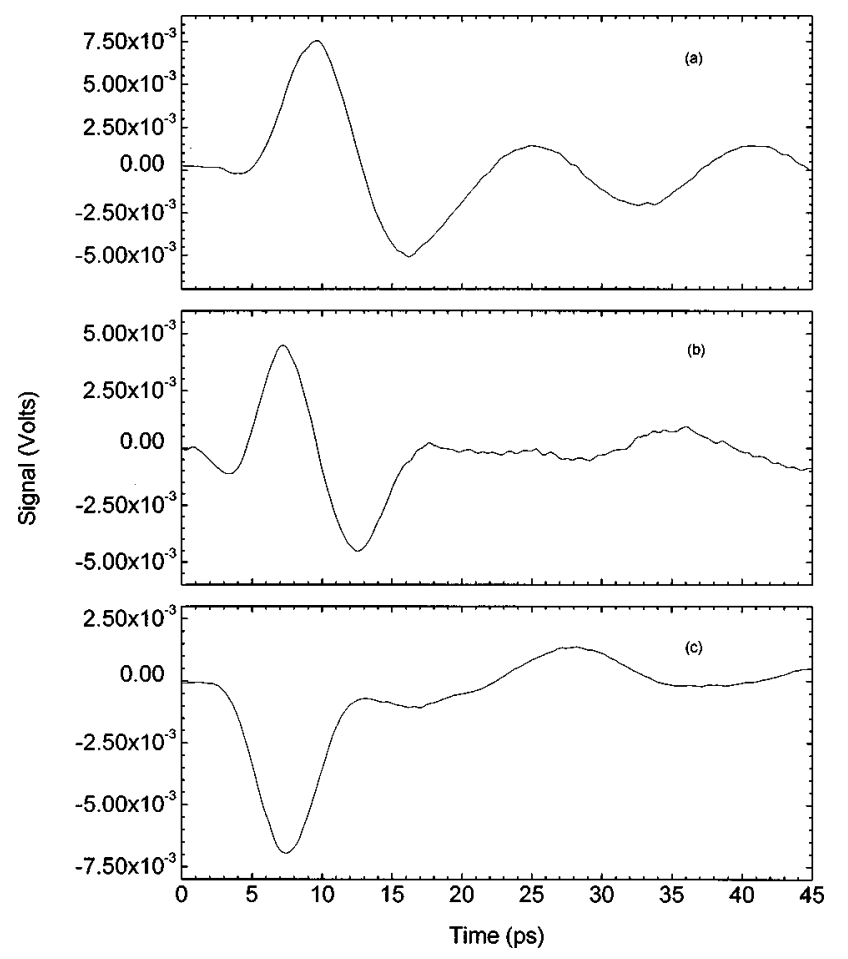

FIG. 3. Temporally-resolved waveforms of the terahertz signal generated by the emitter. Measurements were made using a fiber-mounted epitaxial liftoff LT-GaAs photoconductive probe with an integrated JFET source follower. (a) Measurement made with the setup shown in Fig. 2 with the photoconductive probe $500 \mu \mathrm{m}$ away from the emitter in the $-x$ direction. (b) Measurement taken with the photoconductive probe placed several tens of microns away from the emitter in the $+z$ direction (c) Measurement made with the photoconductive probe positioned $500 \mu \mathrm{m}$ away from the emitter in the $+x$ direction.

ture behaves as a magnetic dipole, allowing radiation to be transmitted planar to the PC switch area.

To explain the increased magnetic dipole radiation relative to the electric dipole, a simple dipole model is used. The electric field $\mathbf{E}_{e}(r, t)$ due to an electric simple or hertzian dipole is

$$
\mathbf{E}_{e}(r, t)=\frac{\mu_{0} s_{d}}{4 \pi r} \frac{\partial i_{e}(t)}{\partial t}(\cos \theta \sin \phi \hat{\theta}+\cos \phi \hat{\phi}),
$$

where $i_{e}(t)$ is the current across the dipole, $\mu_{0}$ is the freespace permeability, $s_{d}$ is the length of the electric dipole, $\hat{\phi}, \hat{\theta}$ are unit vectors in the $\phi, \theta$ direction (Fig. 2), and $r$ is the distance to the center of the dipole. The electric field due to magnetic dipole radiation is given by

$$
\mathbf{E}_{m}(r, t)=-\frac{\mu_{0} s_{m}^{2}}{4 \pi c r} \frac{\partial^{2} i_{m}(t)}{\partial t^{2}} \sin \theta \hat{\phi},
$$

where $i_{m}(t)$ is the current in the magnetic dipole, $s_{m}^{2}$ is the area of magnetic wire loop with length $s_{m}$, and $c$ is the speed of light. Both relationships assume that the dimensions $s_{d}$ and $s_{m}$ are small compared to both $r$ and $\lambda$, the wavelength components of the frequency spectrum. Also, a radiation zone approximation where $r \gg \lambda$ is assumed. In order to compare the far-field contributions of the two dipoles, a Fourier transform is performed on Eqs. (1) and (2). The ratio of the magnetic dipole field to the electric dipole field in the $x-y$ plane is $s_{m}^{2} \omega / s_{d} c$, where $\omega$ is the frequency of the signal. Typically, for electric and magnetic dipoles of the same 


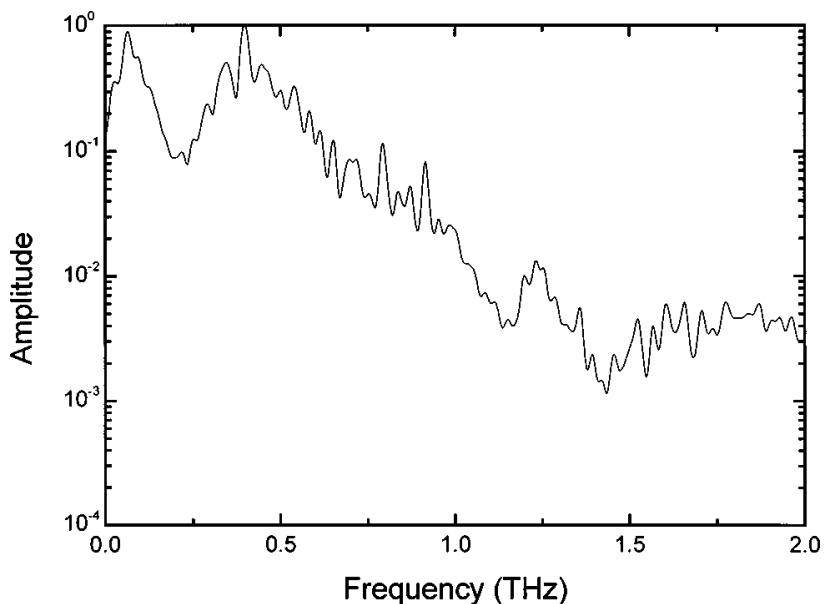

FIG. 4. Amplitude spectrum of the terahertz pulse due to the magnetic dipole generated by the emitter.

dimensions the electric dipole radiation dominates, but as the size of the magnetic dipole becomes significantly larger, as in the case of our emitter, the magnetic dipole contribution becomes important. Although the small dipole approximation fails since $s_{m} \sim \lambda$, and the emitter electrode is not a circular loop, the analysis gives a qualitative explanation for the increased field in the $\phi$ direction.

The signal due to the electric dipole was measured by placing the emitter only $50 \mu \mathrm{m}$ above the PC probe in the $z$ direction with their surfaces parallel to each other [Fig. 3(b)]. An oscillating signal with a $16 \mathrm{ps}$ period is observed after the initial peak for the magnetic dipole signal [Fig. 3(a)] but not for the electric dipole signal. The oscillations found for the magnetic dipole are probably the result of inductance in the looping antenna structure and the capacitance of the switch.

Signals were also compared at two positions in the plane of the emitter on opposite sides of the PC switch. Figure 3(a) shows the signal when the PC probe was placed $500 \mu \mathrm{m}$ away from the ground-electrode side of the emitter. Figure 3(c) shows the signal when the PC probe is positioned 500 $\mu \mathrm{m}$ away from the PC switch side of the emitter. The opposite polarities of the measured waveforms further confirm that the radiation is mostly due to the magnetic dipole, since the field is the $-\hat{\phi}$ direction in the $x-y$ plane. A strong effect of the electric dipole can be ruled out because it would have produced, at the two positions, a field in the same direction according to Eq. (1). Ignoring polarity, differences between the two waveforms can be attributed to the asymmetry of the emitter, with the PC switch on one side of the apex of the metal electrodes allowing some contribution of the electric field dipole due to a closer proximity of the detector.

Because the waveform is a correlation of the $\mathrm{THz}$ signal and the demonstrated $4.5 \mathrm{ps}$ response of this PC probe, the measurement technique limits the bandwidth measured to $<1 \mathrm{THz}$. By applying a Fourier transform and dividing the waveform spectrum by the photoconductive-probe response spectrum, the amplitude spectrum of the radiated signal due to the magnetic dipole was found (Fig. 4). The signal oscillations, which continued for a least five cycles, can account for the low frequency components of the spectrum.

In conclusion, micromachining technology has enabled the fabrication of a freely positionable point source emitter of terahertz radiation. The simple setup does not require any bulk optical components. Because of the electrode geometry used to provide an efficient, ultrasmall $\mathrm{THz}$ emitter, a magnetic dipole component of the radiation exists as well as an electric dipole component.

This work was supported by the National Science Foundation through the Center for Ultrafast Optical Science under STCPHY8920108, and the Air Force Office of Scientific Research, Air Force Materiel Command, USAF, under Grant No. DOD-G-F49620-95-1-0227.

${ }^{1}$ M. C. Nuss, K. W. Goosen, J. P. Gordon, P. M. Mankiewich, M. L. O'Malley, and M. Bhushan, J. Appl. Phys. 70, 2238 (1991).

${ }^{2}$ D. Some and A. V. Nurmikko, Appl. Phys. Lett. 65, 3377 (1994).

${ }^{3}$ D. Grischkowsky, S. R. Keiding, M. Van Exter, and Ch. Fattinger, J. Opt. Soc. Am. 7, 2006 (1990).

${ }^{4}$ J. Son, T. B. Norris, and J. F. Whitaker, J. Opt. Soc. Am. B 11, 2519 (1994).

${ }^{5}$ D. M. Mittleman, R. H. Jacobsen, and M. C. Nuss, IEEE J. Sel. Top. Quantum Electron. 2, 679 (1996).

${ }^{6}$ P. R. Smith, D. H. Auston, and M. C. Nuss, IEEE J. Quantum Electron. 24, 255 (1988).

${ }^{7}$ R. K. Lai, J. R. Hwang, T. B. Norris, and J. F. Whitaker, Appl. Phys. Lett. 69, 1843 (1996).

${ }^{8}$ J. Nees, S. Wakana, and S. Hama, Opt. Quantum Electron. 28, 243 (1996). 